\title{
Prognostic implications of glucose-lowering treatment in patients with acute myocardial infarction and diabetes: experiences from an extended follow-up of the Diabetes Mellitus Insulin-Glucose Infusion in Acute Myocardial Infarction (DIGAMI) 2 Study
}

\author{
L. G. Mellbin • K. Malmberg • A. Norhammar • \\ H. Wedel • L. Rydén • for the DIGAMI 2 Investigators
}

Received: 3 August 2010 / Accepted: 6 January 2011 / Published online: 26 February 2011

(C) Springer-Verlag 2011

\begin{abstract}
Aims/hypothesis This post hoc analysis from the Diabetes Mellitus Insulin-Glucose Infusion in Acute Myocardial Infarction (DIGAMI) 2 trial reports on extended long-term outcome in relation to glucose-lowering agents in patients with myocardial infarction and type 2 diabetes.

Methods Patients were randomised as follows: group 1, insulin-based treatment; group 2, insulin during hospitalisation followed by conventional glucose control; and group 3, conventional treatment. Treatment according to the above protocol lasted 2.1 years. Using the total DIGAMI 2 cohort as an epidemiological database, this study presents mortality rates in the randomised groups, and mortality and morbidity rates by glucose-lowering treatment during an extended period of follow-up (median 4.1 and max 8.1 years).

Results Follow-up data were available in 1,145 of the 1,253 patients. The mortality rate was $31 \%$ ( $72 \%$ cardiovascular) without significant differences between treatment groups. The total number of fatal malignancies was 37 , with a trend towards a higher risk in group 1. The HR for death from malignant disease, compared with group 2, was 1.77 (95\%
\end{abstract}

Electronic supplementary material The online version of this article (doi:10.1007/s00125-011-2084-x) contains supplementary material, which is available to authorised users.

L. G. Mellbin $(\varangle) \cdot K$. Malmberg $\cdot$ A. Norhammar $\cdot$ L. Rydén Cardiology Unit, Department of Medicine, Karolinska Institutet, 17176 Stockholm, Sweden

e-mail: linda.mellbin@karolinska.se

H. Wedel

Nordic School of Public Health,

Göteborg, Sweden
CI $0.87-3.61 ; p=0.11)$ and $3.60(95 \%$ CI $1.24-10.50$; $p=0.02)$ compared with group 3. Insulin treatment was associated with non-fatal cardiovascular events (OR 1.89 95\% CI $1.35-2.63 ; p=0.0002$ ), but not with mortality (OR $1.30,95 \%$ CI $0.93-1.81 ; p=0.13)$. Metformin was associated with a lower mortality rate (HR $0.65,95 \%$ CI $0.47-$ $0.90 ; p=0.01)$ and a lower risk of death from malignancies (HR 0.25, 95\% CI 0.08-0.83; $p=0.02$ ).

Conclusions/interpretation Patients with type 2 diabetes and myocardial infarction have a poor prognosis. Glucoselowering drugs appear to be of prognostic importance. Insulin may be associated with an increased risk of nonfatal cardiac events, while metformin seems to be protective against risk of death.

Keywords Cardiac complications - Clinical diabetes . Insulin therapy · Macrovascular disease - Malignancies . Metformin · Oral pharmacological agents

\section{Abbreviation}

DIGAMI Diabetes Mellitus Insulin-Glucose Infusion in Acute Myocardial Infarction

\section{Introduction}

Despite efforts to decrease macrovascular complications and their prognostic implication in patients with diabetes there are still unresolved issues. Patients with diabetes and coronary artery disease benefit from evidence-based treatment such as statins, platelet-stabilising drugs, betablockers and revascularisation to an even greater degree 
than patients who are free from diabetes [1-3]. In contrast, when it comes to hyperglycaemia, 'the hallmark of diabetes', uncertainties remain. Diagnostic glucose levels are based on the risk of microvascular complications [4], but treatment targets for the prevention of macrovascular disease and best management to reach these targets are still being debated, especially in patients with established macrovascular complications $[5,6]$.

Recent studies have indicated that not only glucose control as such, but also how it is accomplished, i.e. the rapidity of agent action and which class of drugs to use, impact on the prognosis. Some glucose-lowering drugs, for example thiazolidinediones, have been related to negative cardiovascular effects, in particular those increasing the risk of heart failure [7-10]. Exogenous insulin has been related to an increased risk of malignancies in some [11, 12], but not all $[13,14]$ studies, while metformin has been claimed to be protective in this respect. These data are mainly based on registries and are accordingly in need of further confirmation.

In the first Diabetes Mellitus Insulin-Glucose Infusion in Acute Myocardial Infarction (DIGAMI 1) Study [15], an insulin-based compared with conventional glucoselowering management improved survival in patients with acute myocardial infarction and type 2 diabetes. The benefit of insulin treatment was not confirmed in the second DIGAMI trial (DIGAMI 2) [16]. In the latter study, patients with type 2 diabetes and acute myocardial infarction were randomised to three different glucoselowering strategies with no significant differences in the total mortality or cardiovascular morbidity rates between treatment strategies. The interpretation of the discrepant outcomes of DIGAMI 1 and 2 was that, in contrast with the first study, there was no difference in glucose control between the treatment arms in the second trial. Indeed, there was a trend towards diverging mortality and morbidity rates to the benefit of patients without insulin treatment by the end of DIGAMI 2. A subsequent report from this trial [17], focusing on the actual glucoselowering treatment, reported a higher rate of non-fatal reinfarction and stroke among patients receiving insulin. There was also a higher rate of death due to malignancies in the group randomised to insulin [16]. These findings prompted investigators to study whether the outcome pattern would be sustained after a longer period of followup, not the least in light of recent concerns regarding some glucose-lowering drugs.

The aim of the present post hoc exploratory analysis of an extended follow-up of the DIGAMI 2 trial was to report on mortality rates in the original, randomised groups and mortality and morbidity in relation to glucose-lowering agents, using the total cohort as an epidemiological database.

\section{Methods}

\section{Patients}

DIGAMI 2, a prospective, randomised open trial with blinded evaluation, compared three different management strategies in 1,253 patients with type 2 diabetes and suspected acute myocardial infarction [16]. The inclusion criteria were type 2 diabetes (established or admission blood glucose $>11.0 \mathrm{mmol} / \mathrm{l}$ ) and hospitalisation due to suspected acute myocardial infarction on the basis of chest pain $>15$ min during the preceding $24 \mathrm{~h}$ and/or recent ECG signs (new Q-waves and/or ST-segment deviations in two or more leads). Exclusion criteria were inability to cope with insulin treatment or to receive information on the study, residence outside the hospital catchment area, participation in other studies or previous participation in DIGAMI 2.

Of the included patients, $84 \%$ fulfilled the diagnosis of myocardial infarction and almost all remaining patients had coronary artery disease, mostly presenting as unstable angina pectoris.

When the original study ended in December 2003, all centres were asked for additional information on their DIGAMI 2 patients for the period until December 2005. Details requested included vital status, cause and date of death when applicable, and occurrence of myocardial infarction or stroke (yes/no). Data were collected by a case record form submitted to the central study office, accompanied by relevant extracts from hospital records and death certificates. Long-term follow-up data were available in 1,145 patients $(91 \%)$ of the original cohort $(n=1,253)$. These patients were distributed in the original treatment groups as follows: group 1, 431 patients $(91 \%$ of all patients in group 1); group 2, 441 patients (93\%); and group 3, 273 patients (89\%). A diagram, explaining the composition of the different patient cohorts is presented in Fig. 1.

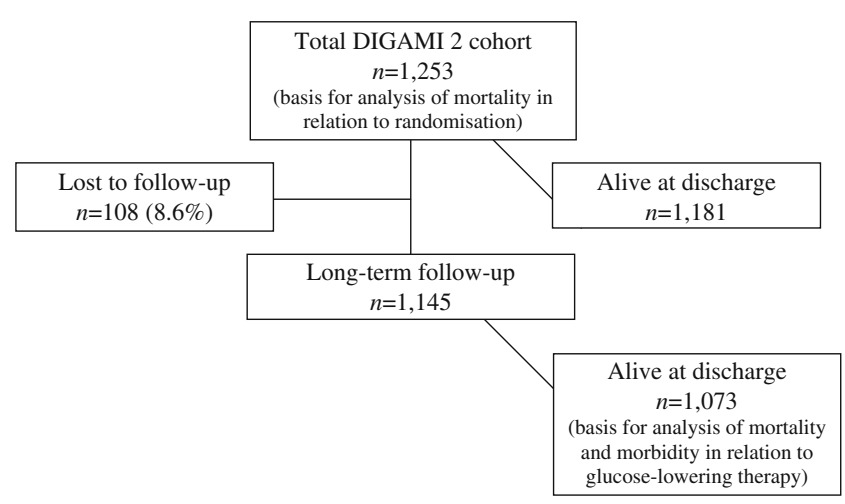

Fig. 1 Flow diagram of how the different groups of patients were selected 


\section{Treatments}

Glucose-lowering treatment The patients were randomised to one of three study arms receiving treatments as follows. Group 1 received insulin-glucose infusion [15], which was intended to decrease blood glucose as fast as possible and keep it between 7 and $10 \mathrm{mmol} / \mathrm{l}$. The infusion lasted until stable normoglycaemia and at least for $24 \mathrm{~h}$, and was followed by subcutaneous insulin-based long-term glucose control. Insulin was administered as short-acting insulin before meals and intermediate long-acting insulin in the evening with a treatment goal of fasting blood glucose 5 to $7 \mathrm{mmol} / \mathrm{l}$ and non-fasting glucose $<10 \mathrm{mmol} / \mathrm{l}$. Group 2 received the same initial treatment as group 1, followed by glucose-lowering treatment according to local practice without a protocol-stated target glucose. Group 3 received glucose-lowering treatment according to local practice.

Concomitant treatment The protocol stated that the use of concomitant treatment should be as uniform as possible and emphasised that all patients without contraindications should receive evidence-based treatment according to international guidelines for acute myocardial infarction $[18,19]$.

\section{Events}

The original primary endpoint was death [16]. Other outcomes were non-fatal reinfarction and stroke. During the original period of follow-up all events were verified by an independent Adjudication Committee. Events during the extended follow-up were adjudicated by two of the present investigators (L. Rydén, L. G. Mellbin) by scrutinising copies of relevant parts of the hospital records and/or death certificates. Both investigators were blinded to treatment group status of patients.

\section{Ethical considerations}

The study conformed to good clinical practice guidelines and followed the recommendations of the Helsinki Declaration. Local Ethics Review Boards approved the DIGAMI 2 protocol. Written informed consent was obtained from all patients prior to enrolment.

\section{Statistical methods}

For comparison between randomised groups the $\chi^{2}$ test was used for non-ordered categorical variables. The Cox proportional hazards model was the basis for analysing mortality and is presented as HR and 95\% CI.

To make the relation between mortality counts and follow-up time more transparent, Kaplan-Meier mortality estimates at a fixed time point (5 years after randomisation) are presented in Table 1. Crude mortality rates are presented in electronic supplementary material (ESM) Table 1.

Logistic regression analysis was used for non-fatal events since the dates of these events were unavailable during the period of extended follow-up. The accuracy of logistic analysis was verified vs the Cox proportional hazard model for mortality rates. The effects of insulin treatment on non-fatal and fatal events, as presented, are based on results from the logistic analysis. In the multivariable models predicting endpoints, the following covariates were adjusted for: sex, age, smoking habits, previous myocardial infarction or previous congestive heart failure recorded at time of hospital admission, creatinine at randomisation, percutaneous transluminal coronary angioplasty or coronary artery bypass grafting during hospitalisation, and blood glucose at randomisation. These were selected as those remaining as significant covariates in multivariable models predicting endpoints in the previous report on the impact of glucose-lowering treatment in

Table 1 Mortality rates and specific causes of death during the extended follow-up

\begin{tabular}{|c|c|c|c|}
\hline Cause of death & $\begin{array}{l}\text { Group 1 } \\
(n=474)\end{array}$ & $\begin{array}{l}\text { Group 2 } \\
(n=473)\end{array}$ & $\begin{array}{l}\text { Group } 3 \\
(n=306)\end{array}$ \\
\hline All deaths ${ }^{\mathrm{a}}$ & $153(0.34)$ & $147(0.32)$ & $89(0.32)$ \\
\hline Cardiovascular deaths $^{\mathrm{b}}$ & $104(0.24)$ & $115(0.25)$ & $59(0.20)$ \\
\hline Myocardial infarction & $53(0.13)$ & $61(0.14)$ & $27(0.09)$ \\
\hline Sudden cardiac death & $29(0.07)$ & $28(0.07)$ & $20(0.08)$ \\
\hline Stroke & $9(0.02)$ & $6(0.01)$ & $4(0.01)$ \\
\hline Heart failure & $9(0.02)$ & $21(0.06)$ & $9(0.04)$ \\
\hline Other cardiovascular & $6(0.02)$ & $6(0.01)$ & $2(0.01)$ \\
\hline Non-cardiovascular deaths ${ }^{\mathrm{c}}$ & $38(0.09)$ & $21(0.06)$ & $17(0.10)$ \\
\hline Malignancies $^{\mathrm{d}}$ & $21(0.05)$ & $12(0.04)$ & $4(0.02)$ \\
\hline Other non-cardiovascular & $17(0.04)$ & $9(0.02)$ & $13(0.08)$ \\
\hline Unknown cause of death & $11(0.04)$ & $11(0.03)$ & $13(0.06)$ \\
\hline
\end{tabular}

Values are categorical variables and expressed as $n$ (Kaplan-Meier 5 year estimate)

${ }^{\text {a }}$ Cox regression analyses for all deaths: group 1 vs group 2 HR 1.05 (95\% CI $0.84-1.31), p=0.69$; group 1 vs group $3 \mathrm{HR} 1.17(95 \% \mathrm{CI}$ $0.90-1.52), p=0.24$; group 2 vs group 3 HR 1.12 (95\% CI 0.86-1.46), $p=0.41$

${ }^{\mathrm{b}}$ Cox regression analyses for cardiovascular deaths: group 1 vs group 2 HR 0.91 (95\% CI $0.70-1.18), p=0.47$; group 1 vs group 3 HR 1.19 (95\% CI 0.86-1.64), $p=0.29$; group 2 vs group $3 \mathrm{HR} 1.32$ (95\% CI $0.97-1.81), p=0.08$

${ }^{\mathrm{c}}$ Cox regression analyses for non-cardiovascular deaths: group 1 vs group 2 HR 1.84 (95\% CI 1.08-3.13), $p=0.03$; group 1 vs group $3 \mathrm{HR}$ 1.55 (95\% CI $0.88-2.75), p=0.13$; group 2 vs group 3 HR $0.84(95 \%$ CI $0.44-1.59), p=0.59$

${ }^{\mathrm{d}}$ Cox regression analyses for deaths from malignancy: group 1 vs group 2 HR 1.77 (95\% CI 0.87-3.61), $p=0.11$; group 1 vs group $3 \mathrm{HR}$ 3.60 (95\% CI 1.24-10.50), $p=0.02$; group 2 vs group 3 HR $2.01(95 \%$ CI $0.65-6.22), p=0.23$ 
patients discharged from hospital [17]. In the logistic regression models, metformin was added as a covariate. Body weight (BMI) was not a significant risk factor for fatal or non-fatal events [20] and was not therefore included as a confounder.

In the Forest plot (Fig. 2), glucose-lowering agent (i.e. insulin, metformin and sulfonylurea) and blood glucose were used as time-dependent (updated) variables in a Cox timedependent model. The use of drugs was updated at each visit until the last one, which either preceded an event or was the close-out visit at the end of the initial period of follow-up. In the present study, updated information is denoted 'most recent exposure to a drug'. This ascertained that drugs added or excluded during the period from hospital discharge to the end of 2003 were taken into account when looking at the impact of therapy. During the extended follow-up the last updated value from the close-out visit was kept constant, since no further information was available on glucose values or changes in treatment. Updated blood glucose was expressed as the average of all glucose values from the time of hospital discharge until the end of the original follow-up. No interaction terms were used due to limited power.

Kaplan-Meier curves for mortality, using randomised treatment groups as strata, are presented for illustration purposes $(p$ value $=\log$-rank test for trend; Fig. 3). Since multiple comparisons were performed, the two-sided $p$ value for significance was adjusted to 0.025. All statistical analyses were performed by SAS version 9.2 (SAS Institute, Cary, NC, USA).

\section{Results}

Patient population

The baseline characteristics of the original cohort of 1,253 patients have been presented in the original

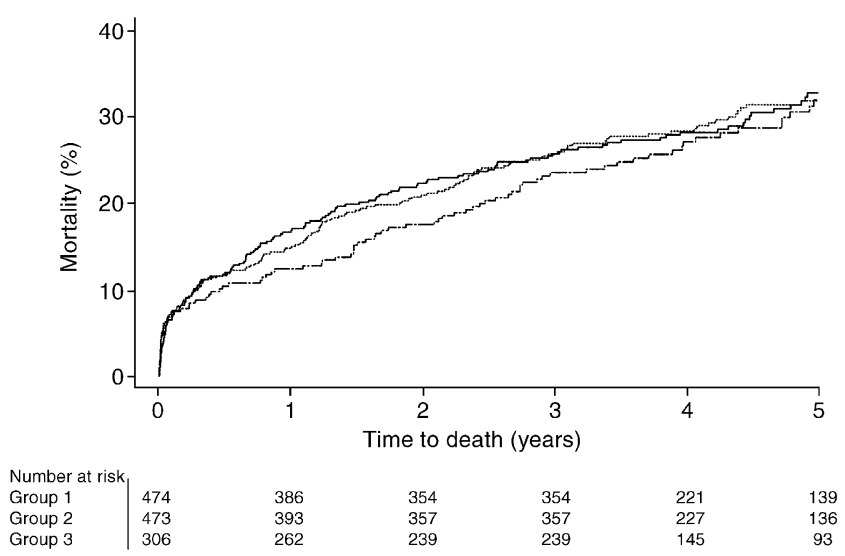

Fig. 2 Total mortality rate in groups 1, 2 and 3; $p=0.47$ (log rank test for trend). Continuous line, group 1; dotted line, group 2; dashed line, group 3 publication [16]. Table 2 presents baseline characteristics, including pertinent biochemical and clinical data, at the time of randomisation and at discharge for patients who survived the hospital phase and for whom long-term follow-up data were available $(n=1,073)$. Patients were followed for a maximum of 8.3 years (median 4.1; interquartile range 2.6-5.4).

The distribution of glucose-lowering treatment at discharge and at the last visit in the study, including the last visit preceding death for deceased patients, is outlined in Table 3. During the study period, the use of insulin decreased in group 1 and increased in groups 2 and 3 . The use of oral glucose-lowering drugs increased in all groups, most noticeably in group 1 with a doubling in the use of metformin. By the end of the original follow-up, $13.4 \%$ were not receiving any glucose-lowering agent, with the highest percentage in group 3.

Mortality rates in relation to randomisation

Of the 1,253 participants randomised, 389 (31\%) patients died, the majority 278 (22\%) of cardiovascular causes (Table 1, ESM Table 1). Cox regression analysis did not show any difference in total or cardiovascular mortality rates between the three groups, although non-cardiovascular deaths were more common in group 1 than group 2, HR 1.84 (95\% CI 1.08-3.13; $p=0.03)$. The Kaplan-Meier curves for total mortality rate by group from randomisation are presented in Fig. 2.

The total number of deaths due to malignant diseases was $37(9.5 \%$ of all deaths). The risk was highest in patients randomised to long-term insulin (group 1). The HR comparing groups 1 and 2 was 1.77 (95\% CI 0.87-3.61; $p=0.11)$ and $3.60(95 \%$ CI $1.24-10.50 ; p=0.02)$ comparing groups 1 and 3.

Mortality and morbidity rates in relation to glucose-lowering therapy

In the logistic analysis, insulin treatment was accompanied by a higher risk of non-fatal events (myocardial infarction $n=192$; stroke $n=69$ ) and the combination of fatal and non-fatal events. The adjusted impact of insulin treatment on mortality and morbidity rates in patients discharged alive $(n=1,073)$ is presented in Table 4 . Comparison of all patients on insulin at discharge with patients not on insulin revealed that the former had a higher risk of non-fatal cardiovascular events (OR 1.89, 95\% CI 1.35-2.63; $p=0.0002$ ) but not increased mortality (OR 1.30, 95\% CI $0.93-1.81 ; p=0.13$ ). This finding remained valid when comparing patients who were prescribed new insulin during the index hospitalisation and those without 
Hazard ratio

$(95 \% \mathrm{Cl})$

Metformin (185/888) ${ }^{a}$

Death $(47 / 271)^{\mathrm{b}}$

CV death $(31 / 176)^{\mathrm{b}}$

Cancer death $(6 / 31)^{b}$

Sulfonylurea (250/823)

Death $(79 / 239)^{\mathrm{b}}$

CV death $(57 / 152)^{\text {b }}$

Cancer death $(11 / 26)^{\mathrm{b}}$

Insulin (631/442) ${ }^{\mathrm{a}}$

Death $(208 / 110)^{b}$

CV death $(133 / 74)^{\mathrm{b}}$

Cancer death $(27 / 10)^{\mathrm{b}}$

Any glucose-lowering drug (912/161) ${ }^{\text {a }}$

Death $(276 / 42)^{b}$

CV death $(181 / 26)^{b}$

Cancer death $(33 / 4)^{b}$
$0.65(0.47-0.90)$

$0.72(0.49-1.06)$

$0.25(0.08-0.83)$

$1.09(0.84-1.42)$

$1.29(0.94-1.76)$

$0.67(0.28-1.61)$

$1.03(0.80-1.31)$

$0.90(0.67-1.21)$

$2.05(0.95-4.43)$

$0.83(0.60-1.16)$

$0.79(0.53-1.17)$

$1.01(0.38-2.69)$ $p$-value

0.0094
0.0995
0.0235


0.5251
0.1145
0.3686


0.8312
0.4726
0.0689


0.2737
0.2398
0.9792

0.05

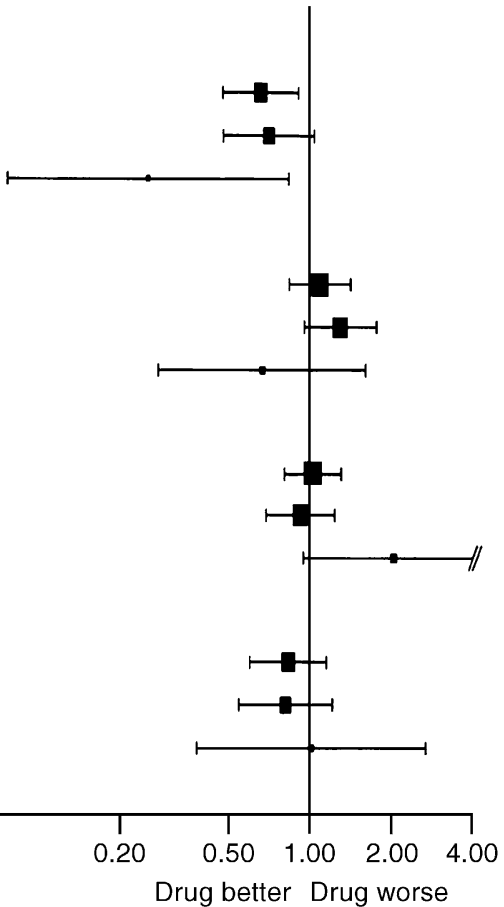

luminal coronary angioplasty or coronary artery bypass grafting during the hospitalisation, and mean updated blood glucose. ${ }^{a}$ Number of patients using drug/number of patients not using drug at discharge; ${ }^{b}$ number of endpoints for patients using drug/number of endpoints for patients not using drug

ended, was to further study the cardiovascular outcomes in these patients. The need for prolonged observation was underlined by the observation that chronic insulin therapy seemed to increase and metformin to decrease the number of non-fatal cardiovascular events, albeit at the time without any impact on mortality rates [17]. Moreover, the original data indicated a higher number of deaths from malignant disease among patients in the original group 1 (long-term insulin), another important reason to extend the observation.

The present cohort should be representative of patients with type 2 diabetes and myocardial infarctions, considering that the DIGAMI 2 trial had very few exclusion criteria, that no patient was lost in the original follow-up and that this report comprises a very high proportion, $91 \%$, of the original population. Moreover, a large proportion of the patients were prescribed evidence-based pharmacological treatment as early as the time of hospital discharge, a therapeutic strategy that increased over time [16]. Updated information on drug exposure was obtained at follow-up visits after 3, 6, 9 and 12 months, and thereafter every 6th month. Since adjustments of glucose-lowering therapy were relatively limited over time, it is reasonable to conclude that the last visit updating accurately reflects long-term exposure.

The increase in mortality rate from $21 \%$ by the end of the initial follow-up [16] to the present $31 \%$ shows not only 
Table 2 Baseline characteristics and medications at admission and discharge for patients who survived the hospital phase and for whom long-term follow-up data were available

\begin{tabular}{|c|c|c|c|c|}
\hline Variable & Total & Group 1 & Group 2 & Group 3 \\
\hline$n$ & 1,073 & 407 & 409 & 257 \\
\hline Age (years) & $68.3(10.9)$ & $68.3(11.2)$ & $68.3(10.5)$ & $68.5(10.8)$ \\
\hline Men, $n(\%)$ & $719(67.0)$ & $271(66.6)$ & $275(67.2)$ & $173(67.3)$ \\
\hline BMI $\left(\mathrm{kg} / \mathrm{m}^{2}\right)$ & $28.3(4.6)$ & $28.2(4.7)$ & $28.4(4.7)$ & $28.5(4.5)$ \\
\hline Diabetes duration (years) & $8.14(8.39)$ & $8.21(8.40)$ & $7.79(8.47)$ & $8.60(8.27)$ \\
\hline \multicolumn{5}{|l|}{ Blood pressure (mmHg) } \\
\hline Systolic & $136.0(25.1)$ & $137.3(25.0)$ & $133.2(23.5)$ & $138.3(27.5)$ \\
\hline Diastolic & $76.4(15.6)$ & $76.8(15.9)$ & $75.4(14.9)$ & $77.5(16.1)$ \\
\hline \multicolumn{5}{|l|}{ Previous medical history, $n(\%)$} \\
\hline Myocardial infarction & $374(34.9)$ & $152(37.3)$ & $146(35.7)$ & $76(29.6)$ \\
\hline Angina pectoris & $491(45.8)$ & $190(46.7)$ & $191(46.7)$ & $110(42.8)$ \\
\hline Chronic congestive heart failure & $202(18.8)$ & $81(19.9)$ & $76(18.6)$ & $45(17.5)$ \\
\hline Hypertension & $537(50.1)$ & $220(54.1)$ & $198(48.5)$ & $119(46.3)$ \\
\hline Hyperlipidaemia & $353(32.9)$ & $133(32.7)$ & $136(33.3)$ & $84(32.7)$ \\
\hline Current smoker & $257(24.1)$ & $84(20.8)$ & $113(27.8)$ & $60(23.4)$ \\
\hline Previous CABG & $125(11.6)$ & $47(11.5)$ & $52(12.7)$ & $26(10.1)$ \\
\hline Previous PTCA & $95(8.9)$ & $39(9.6)$ & $37(9.0)$ & $19(7.4)$ \\
\hline \multicolumn{5}{|l|}{ Medication prior to admission, $n(\%)$} \\
\hline Insulin & $361(33.6)$ & $133(32.7)$ & $144(35.2)$ & $84(32.7)$ \\
\hline Metformin & $266(24.8)$ & $98(24.1)$ & $99(24.3)$ & $69(26.8)$ \\
\hline Glibenclamide & $246(22.9)$ & $107(26.3)$ & $83(20.3)$ & $56(21.8)$ \\
\hline Beta-blockers & $459(42.8)$ & $175(43.0)$ & $181(44.3)$ & $103(40.2)$ \\
\hline Aspirin & $539(50.2)$ & $215(52.8)$ & $195(47.7)$ & $129(50.2)$ \\
\hline ACE inhibitors & $348(32.5)$ & $130(32.0)$ & $130(31.8)$ & $88(34.2)$ \\
\hline Lipid-lowering & $314(29.3)$ & $123(30.2)$ & $122(29.9)$ & $69(26.8)$ \\
\hline \multicolumn{5}{|l|}{ Biochemistry at admission } \\
\hline Blood glucose (mmol/l) & $12.5(4.2)$ & $12.6(4.3)$ & $12.3(4.1)$ & $12.8(4.4)$ \\
\hline $\mathrm{HbA}_{1 \mathrm{c}}(\%)^{\mathrm{a}}$ & $7.68(1.74)$ & $7.59(1.67)$ & $7.70(1.75)$ & $7.80(1.82)$ \\
\hline Serum creatinine $(\mu \mathrm{mol} / \mathrm{l})$ & $102.8(43.0)$ & $101.1(42.2)$ & $105.4(48.6)$ & $101.2(34.1)$ \\
\hline Serum cholesterol (mmol/l) & $5.15(1.25)$ & $5.13(1.21)$ & $5.14(1.29)$ & $5.22(1.25)$ \\
\hline Serum triacylglycerol $(\mathrm{mmol} / \mathrm{l})$ & $2.20(2.00)$ & $2.16(1.68)$ & $2.25(2.01)$ & $2.19(2.40)$ \\
\hline \multicolumn{5}{|l|}{ Medications at discharge, $n(\%)$} \\
\hline Beta blockers & $897(83.8)$ & $338(83.0)$ & $349(85.3)$ & $210(82.4)$ \\
\hline Aspirin & $939(87.7)$ & $358(88.0)$ & $366(89.5)$ & $215(84.3)$ \\
\hline ACE inhibitors & $716(66.9)$ & $278(68.3)$ & $275(67.2)$ & $163(63.9)$ \\
\hline Calcium blockers & $186(17.4)$ & $74(18.2)$ & $67(16.4)$ & $45(17.6)$ \\
\hline Ticlopidin or clopidogrel & $221(20.6)$ & $97(23.8)$ & $81(19.8)$ & $43(16.8)$ \\
\hline Diuretics & $536(50.0)$ & $199(48.9)$ & $215(52.6)$ & $122(47.8)$ \\
\hline Lipid-lowering drugs & $700(65.4)$ & $280(68.8)$ & 277 (67.7) & $143(56.1)$ \\
\hline
\end{tabular}

Values are $n(\%)$ for categorical variables and mean $(\mathrm{SD})$ for continuous variables; $n=1,073$

${ }^{\mathrm{a}} \mathrm{HbA}_{1 \mathrm{c}}$ was analysed by the Mono-S standard with an upper normal limit of $5.3 \%$

CABG, coronary artery bypass grafting; PTCA, percutaneous transluminal coronary angioplasty
The discrepancy between the two DIGAMI trial findings with regard to mortality reduction by insulin-based glycaemic control may relate to the pre-existing conditions at the start of these studies. In DIGAMI 1 [15], admission $\mathrm{HbA}_{1 \mathrm{c}}$ decreased by up to $1.5 \%$ from a high level $(9.1 \%$ recalculated to DCCT standard), representing a considerable improvement of glycaemic control compared with that achieved in DIGAMI 2. In the latter study, glucose control was similar in the three treatment arms and failed to reach the treatment goal. Thus the decrease in $\mathrm{HbA}_{1 \mathrm{c}}$ was only 
Table 3 Glucose-lowering treatment at hospital discharge and last visit (including last visit preceding death) for patients who survived the hospital phase and for whom long-term follow-up data were available

Values are categorical variables and expressed as $n(\%)$

\begin{tabular}{llllll}
\hline Patient $(n)$ and drug per time point & Total & Group 1 & Group 2 & Group 3 & $p$ value \\
\hline$n$ (total) & 1073 & 407 & 409 & 257 & \\
Discharge & & & & & \\
$n$ & & 407 & 409 & 257 & \\
Oral, $n(\%)$ & $397(37.0)$ & $50(12.3)$ & $211(51.6)$ & $136(52.9)$ & $<0.0001$ \\
$\quad$ Sulfonylurea, $n(\%)$ & $250(23.3)$ & $32(7.9)$ & $128(31.3)$ & $90(35.0)$ & $<0.0001$ \\
Metformin, $n(\%)$ & $185(17.2)$ & $24(5.9)$ & $102(24.9)$ & $59(23.0)$ & $<0.0001$ \\
Acarbose, $n(\%)$ & $8(0.7)$ & $0(0.0)$ & $4(1.0)$ & $4(1.6)$ & 0.06 \\
Other, $n(\%)$ & $45(4.2)$ & $1(0.2)$ & $27(6.6)$ & $17(6.7)$ & $<0.0001$ \\
Insulin, $n(\%)$ & $631(59.0)$ & $346(85.0)$ & $185(45.3)$ & $100(39.2)$ & $<0.0001$ \\
None, $n(\%)$ & $161(15.0)$ & $37(9.1)$ & $69(16.9)$ & $55(21.4)$ & $<0.0001$ \\
Last visit & & & & & \\
$n$ & & 407 & 409 & 257 & $144(56.0)$ \\
Oral, $n(\%)$ & $450(42.0)$ & $89(22.0)$ & $217(53.1)$ & $<0.0001$ \\
Sulfonylurea, $n(\%)$ & $236(22.0)$ & $42(10.4)$ & $117(28.6)$ & $77(30.0)$ & $<0.0001$ \\
Metformin, $n(\%)$ & $279(26.1)$ & $56(13.8)$ & $133(32.5)$ & $90(35.0)$ & $<0.0001$ \\
Acarbose, $n(\%)$ & $4(0.4)$ & $0(0.0)$ & $1(0.2)$ & $3(1.2)$ & 0.05 \\
Other, $n(\%)$ & $51(4.8)$ & $3(0.7)$ & $29(7.1)$ & $19(7.4)$ & $<0.0001$ \\
Insulin, $n(\%)$ & $642(60.0)$ & $328(81.2)$ & $202(49.4)$ & $112(43.6)$ & $<0.0001$ \\
None, $n(\%)$ & $143(13.4)$ & $40(9.9)$ & $57(13.9)$ & $46(17.9)$ & 0.0114 \\
\hline & & & & &
\end{tabular}

modest (from $8.3 \%$ at admission to $7.8 \%$ by the end of the trial). Considering the findings of two recently published trials, the Action in Diabetes and Vascular Disease: Preterax and Diamicron Modified Release Controlled Evaluation (ADVANCE) trial [7] and the Action to Control Cardiovascular Risk in Diabetes (ACCORD) trial [8], in which mortality rates remained high, despite a more pronounced reduction in $\mathrm{HbA}_{1 \mathrm{c}}$ than in the present study, tighter glucose control of the rather modest hyperglycaemia in DIGAMI 2 can be argued to have been of limited importance for risk of death. This suggests that it may be too late to expect any major benefits from very strict glucose control in patients who already have established serious macrovascular disease manifestations [7, 8]. In contrast, there is some evidence that tight glycaemic control initiated soon after diagnosis of diabetes is associated with long-term reduction of risk of death and myocardial infarction [22-24], especially when combined with multifactorial risk factor management [25]. Observations in patients with myocardial infarction and newly detected glucose disturbances from the Euro Heart Survey on Diabetes and the Heart support this assumption [26].

The extended follow-up of DIGAMI 2 confirms the previous report on increased risk of [17] non-fatal myocardial infarction and stroke in patients on chronic insulin treatment. This finding remained valid in a separate analysis including only patients with newly instituted insulin therapy at discharge, with a majority randomised to such treatment, but it did not translate into an increased mortality rate. It is possible that even longer periods of follow-up are needed until such an effect becomes manifest. In contrast to findings on the impact of long-term insulin, the outcome of metformin therapy was more favourable. In the previous report from DIGAMI 2 [17] on the impact of glucose-lowering therapy, it was noted that metformin treatment reduced the number of non-fatal cardiovascular events (myocardial infarction and stroke), albeit at the time, not that of deaths. Continued observation revealed a significantly lower mortality rate in metformin-treated patients, consistent with other observations of cardiovascular benefits of metformin [24, 27]. In this context, recent observations by Kooy et al. [28] that metformin added to insulin beneficially influenced body weight, glycaemic control and insulin requirement, and also reduced the risk of macrovascular events during a follow-up of somewhat more than 4 years are of interest.

Although the total mortality rate was similar in the three randomised treatment groups, patients randomised to insulin (group 1) had a higher risk of dying of malignant diseases than those randomised to non-insulin-based glucose-lowering therapy (group 3). Although the number of such deaths was small, the difference reached statistical significance. A separate analysis of the impact of long-term insulin treatment revealed a trend towards a higher rate of deaths in malignant conditions, even after adjustment for various covariates. In contrast, patients on metformin had a significantly lower mortality rate from malignancies. Interestingly, the relationship for metformin to cancer was similar with or without insulin as a covariate. This provides added evidence of a potentially preventive effect on cancer 
Table 4 The effect of insulin treatment from the time of hospital discharge for patients discharged alive $(n=1073)$

\begin{tabular}{|c|c|c|}
\hline Patients on insulin & OR $(95 \% \mathrm{CI})$ & $p$ value \\
\hline \multicolumn{3}{|l|}{ Mortality } \\
\hline Insulin $^{\mathrm{a}}$ & $1.30(0.9-1.81)$ & 0.1292 \\
\hline New on insulin ${ }^{b}$ & $1.00(0.65-1.55)$ & 0.9921 \\
\hline \multicolumn{3}{|l|}{ CV death } \\
\hline Insulin $^{\mathrm{a}}$ & $1.15(0.79-1.67)$ & 0.4616 \\
\hline New on insulin ${ }^{b}$ & $1.06(0.65-1.74)$ & 0.8043 \\
\hline \multicolumn{3}{|l|}{ Reinfarction } \\
\hline Insulin $^{a}$ & $1.94(1.34-2.81)$ & 0.0004 \\
\hline New on insulin ${ }^{b}$ & $2.04(1.29-3.21)$ & 0.0021 \\
\hline \multicolumn{3}{|l|}{ Reinfarction/stroke } \\
\hline Insulin $^{\mathrm{a}}$ & $1.89(1.35-2.63)$ & 0.0002 \\
\hline New on insulin ${ }^{b}$ & $2.12(1.40-3.21)$ & 0.0004 \\
\hline \multicolumn{3}{|c|}{ Death/reinfarction/stroke } \\
\hline Insulin $^{\mathrm{a}}$ & $1.78(1.32-2.38)$ & 0.0001 \\
\hline New on insulin ${ }^{\mathrm{b}}$ & $1.65(1.14-2.40)$ & 0.0086 \\
\hline
\end{tabular}

Values are after adjustment for: use of metformin, sex, age and smoking habits, previous myocardial infarction, previous chronic congestive heart failure, creatinine at randomisation, percutaneous transluminal coronary angioplasty during hospitalisation or before randomisation, coronary artery bypass grafting during hospitalisation or before randomisation, and blood glucose at randomisation

Data are presented for all patients on insulin and for those with newly instituted insulin

a $n=631$ patients on insulin at discharge vs $n=442$ patients without insulin at discharge

${ }^{\mathrm{b}} n=272$ patients on insulin at discharge and not on insulin prior to hospitalisation vs $n=440$ patients with no insulin at discharge and no insulin prior to hospitalisation

by metformin. For insulin, the risk of cancer was attenuated when metformin was added as a covariate. Accordingly, it is possible that the supposed cancer risk from insulin may, in fact, be a loss of the beneficial effect of metformin, rather than an adverse effect of insulin as such. Nevertheless, a potential risk of cancer risk with insulin cannot be excluded and future studies should explore this important suspicion. This question could have been further elucidated if it had been possible to do an interaction analysis. However, to be informative, such an analysis would have required a sample size at least four times greater than the present one.

The trend towards a possibility that insulin may be associated with mortality rates from malignancies underlines findings in a recently presented registry study comprising 62,809 patients with diabetes in a UK general practice database. Patients on insulin or insulin secretagogues were more likely to develop solid cancers than those on metformin. Adding metformin to insulin or to sulfonylurea reduced the risk of cancer [12]. It has been suggested that high levels of endogenous insulin, which are common in disorders associated with insulin resistance, such as diabetes and cancer, may impact on the insulinIGF-1 axis. Thus high insulin levels, at least endogenous but possibly also exogenous insulin, may alter growth signals and cause resistance to apoptosis, predisposing to survival and proliferation of malignant cells [29-31]. Metformin has been reported to be protective against cancer, for example in a recent observational cohort study from Scotland [32] and in a population-based cohort study using administrative databases from Saskatchewan Health [31]. The beneficial effects of metformin are thought to be mediated via the AMP-activated protein kinase signalling pathway, which plays an important role in cellular growth and energy balance $[33,34]$. The present report does not give a clear answer to the question of whether insulin induces cancer or promotes proliferation of already existing malignant cells. Furthermore, since the effects of different glucose-lowering drugs are compared with each other, it is not possible to distinguish whether the observed differences between insulin and metformin are an expression of the negative effects of insulin or of a beneficial effect of metformin. A cautious interpretation of the present data supports a beneficial effect of metformin rather than a negative effect of insulin, although the latter must be kept in mind pending further clarification.

\section{Limitations}

The present observation regarding death from malignancies should be treated with due caution. In this context, it should be stressed that patients were not randomised to metformin, and interaction analyses could not be performed due to the small number of events, which also made it impossible to relate mortality rates from malignant conditions to the type of insulin used. Information on non-fatal malignancies would have been useful for further consolidation of the findings. However, an impact on mortality rates from malignancies was an unexpected finding, and detailed information on non-fatal malignant conditions was therefore not a prespecified endpoint in the DIGAMI 2 trial. As already discussed, the data are supported by other reports of similar observations. There are also pathophysiological reasons for assuming that insulin may be harmful and metformin beneficial in this respect. The importance of further study on the relation between different glucoselowering agents and malignant conditions is therefore clearly underlined.

\section{Conclusion}

Mortality remains high in patients with type 2 diabetes and myocardial infarction, despite efforts to achieve good glycaemic control and a strong emphasis on use of 
evidence-based pharmacological treatment. The drug used for glucose control appears to have an impact on prognosis. Insulin treatment, in particular chronic use, seems to be associated with an increased risk of non-fatal cardiac events and possibly even death due to malignancies, while metformin seems to be protective. These observations cannot be regarded as conclusive, but should encourage further testing in prospective studies, i.e. should be seen as hypotheses-generating.

Acknowledgements The authors wish to thank M. Molin and R. Binisi for valuable support with the database. DIGAMI 2 was supported by the Swedish Heart-Lung Foundation, AFA Insurance and by unconditional research grants from Aventis Sweden and Novo Nordisk Denmark.

Duality of interest $\mathrm{K}$. Malmberg is employed part-time by $\mathrm{F}$. Hoffmann-La Roche, which was not involved in the trial and has no interest in this manuscript. The other authors declare that there is no duality of interest associated with this manuscript.

\section{References}

1. Ryden L, Standl E, Bartnik M et al (2007) Guidelines on diabetes, pre-diabetes, and cardiovascular diseases: executive summary. The Task Force on Diabetes and Cardiovascular Diseases of the European Society of Cardiology (ESC) and of the European Association for the Study of Diabetes (EASD). Eur Heart J 28:88-136

2. Anselmino M, Malmberg K, Ohrvik J, Ryden L (2008) Evidencebased medication and revascularization: powerful tools in the management of patients with diabetes and coronary artery disease: a report from the Euro Heart Survey on diabetes and the heart. Eur J Cardiovasc Prev Rehabil 15:216-223

3. Gaede P, Lund-Andersen H, Parving HH, Pedersen O (2008) Effect of a multifactorial intervention on mortality in type 2 diabetes. N Engl J Med 358:580-591

4. [No authors listed] (2006) Definition and diagnosis of diabetes mellitus and intermediate hyperglycemia: report of a WHO/IDF consultation. WHO, Geneva

5. Skyler JS, Bergenstal R, Bonow RO et al (2009) Intensive glycemic control and the prevention of cardiovascular events: implications of the ACCORD, ADVANCE, and VA diabetes trials: a position statement of the American Diabetes Association and a scientific statement of the American College of Cardiology Foundation and the American Heart Association. Circulation 119:351-357

6. Ryden L, Malmberg K, Mellbin L (2009) Is glucose normalization an evidence-based treatment for patients with type 2 diabetes mellitus? Nat Clin Pract Endocrinol Metab 5:8-9

7. Patel A, MacMahon S, Chalmers J et al (2008) Intensive blood glucose control and vascular outcomes in patients with type 2 diabetes. N Engl J Med 358:2560-2572

8. Gerstein HC, Miller ME, Byington RP et al (2008) Effects of intensive glucose lowering in type 2 diabetes. $\mathrm{N}$ Engl $\mathrm{J}$ Med 358:2545-2559

9. Singh S, Loke YK, Furberg CD (2007) Long-term risk of cardiovascular events with rosiglitazone: a meta-analysis. Jama 298:1189-1195

10. Lincoff AM, Wolski K, Nicholls SJ, Nissen SE (2007) Pioglitazone and risk of cardiovascular events in patients with type 2 diabetes mellitus: a meta-analysis of randomized trials. Jama 298:1180-1188
11. Hemkens LG, Grouven U, Bender R et al (2009) Risk of malignancies in patients with diabetes treated with human insulin or insulin analogues: a cohort study. Diabetologia 52: $1732-1744$

12. Currie CJ, Poole CD, Gale EA (2009) The influence of glucoselowering therapies on cancer risk in type 2 diabetes. Diabetologia 52:1766-1777

13. Jonasson JM, Ljung R, Talback M, Haglund B, Gudbjornsdottir S, Steineck G (2009) Insulin glargine use and short-term incidence of malignancies - a population-based follow-up study in Sweden. Diabetologia 52:1745-1754

14. Colhoun HM, SDRN Epidemiology Group (2009) Use of insulin glargine and cancer incidence in Scotland: a study from the Scottish Diabetes Research Network Epidemiology Group. Diabetologia 52:1755-1765, Erratum 52: 2469

15. Malmberg K, Ryden L, Efendic S et al (1995) Randomized trial of insulin-glucose infusion followed by subcutaneous insulin treatment in diabetic patients with acute myocardial infarction (DIGAMI Study): effects on mortality at 1 year. J Am Coll Cardiol 26:57-65

16. Malmberg K, Ryden L, Wedel H et al (2005) Intense metabolic control by means of insulin in patients with diabetes mellitus and acute myocardial infarction (DIGAMI 2): effects on mortality and morbidity. Eur Heart J 26:650-661

17. Mellbin LG, Malmberg K, Norhammar A, Wedel H, Ryden L (2008) The impact of glucose lowering treatment on long-term prognosis in patients with type 2 diabetes and myocardial infarction: a report from the DIGAMI 2 trial. Eur Heart J 29:166-176

18. [No authors listed] (1996) Acute myocardial infarction: prehospital and in-hospital management. The Task Force on the Management of Acute Myocardial Infarction of the European Society of Cardiology. Eur Heart J 17:43-63

19. Van de Werf F, Ardissino D, Betriu A et al (2003) Management of acute myocardial infarction in patients presenting with STsegment elevation. The Task Force on the Management of Acute Myocardial Infarction of the European Society of Cardiology. Eur Heart J 24:28-66

20. Aas AM, Ohrvik J, Malmberg K, Ryden L, Birkeland KI (2009) Insulin-induced weight gain and cardiovascular events in patients with type 2 diabetes. A report from the DIGAMI 2 Study. Diabetes Obes Metab 11:323-329

21. Norhammar A, Lindback J, Ryden L, Wallentin L, Stenestrand U (2007) Improved but still high short- and long-term mortality rates after myocardial infarction in patients with diabetes mellitus: a time-trend report from the Swedish Register of Information and Knowledge about Swedish Heart Intensive Care Admission. Heart 93:1577-1583

22. Nathan DM, Cleary PA, Backlund JY et al (2005) Intensive diabetes treatment and cardiovascular disease in patients with type 1 diabetes. N Engl J Med 353:2643-2653

23. UKPDS33 (1998) Intensive blood-glucose control with sulphonylureas or insulin compared with conventional treatment and risk of complications in patients with type 2 diabetes (UKPDS 33). UK Prospective Diabetes Study (UKPDS) Group. Lancet 352:837-853

24. UKPDS34 (1998) Effect of intensive blood-glucose control with metformin on complications in overweight patients with type 2 diabetes (UKPDS 34). UK Prospective Diabetes Study (UKPDS) Group. Lancet 352:854-865

25. Gaede P, Vedel P, Larsen N, Jensen GV, Parving HH, Pedersen O (2003) Multifactorial intervention and cardiovascular disease in patients with type 2 diabetes. N Engl J Med 348:383-393

26. Anselmino M, Ohrvik J, Malmberg K, Standl E, Ryden L (2008) Glucose lowering treatment in patients with coronary artery disease is prognostically important not only in established but 
also in newly detected diabetes mellitus: a report from the Euro Heart Survey on Diabetes and the Heart. Eur Heart J 29:177-184

27. Bailey CJ (2008) Metformin: effects on micro and macrovascular complications in type 2 diabetes. Cardiovasc Drugs Ther 22: $215-224$

28. Kooy A, de Jager J, Lehert P et al (2009) Long-term effects of metformin on metabolism and microvascular and macrovascular disease in patients with type 2 diabetes mellitus. Arch Intern Med 169:616-625

29. Giovannucci E, Michaud D (2007) The role of obesity and related metabolic disturbances in cancers of the colon, prostate, and pancreas. Gastroenterology 132:2208-2225

30. Holly JM, Perks CM (2008) Cancer as an endocrine problem. Best Pract Res Clin Endocrinol Metab 22:539-550
31. Bowker SL, Majumdar SR, Veugelers P, Johnson JA (2006) Increased cancer-related mortality for patients with type 2 diabetes who use sulfonylureas or insulin. Diabetes Care 29:254-258

32. Libby G, Donnelly LA, Donnan PT, Alessi DR, Morris AD, Evans JM (2009) New users of metformin are at low risk of incident cancer: a cohort study among people with type 2 diabetes. Diabetes Care 32:1620-1625

33. Zakikhani M, Dowling R, Fantus IG, Sonenberg N, Pollak M (2006) Metformin is an AMP kinase-dependent growth inhibitor for breast cancer cells. Cancer Res 66:10269-10273

34. Schimmack G, Defronzo RA, Musi N (2006) AMP-activated protein kinase: role in metabolism and therapeutic implications. Diabetes Obes Metab 8:591-602 